\title{
Les Sours Grises à l’Université de Montréal, 1923-1947 : de la gestion hospitalière à l'enseignement supérieur en nursing ${ }^{1}$
}

\author{
Esther Lamontagne et Yolande Cohen
}

Cet article présente le rôle des Soeurs Grises dans le développement d'un enseignement universitaire du nursing au Québec. À travers le dépouillement de leurs archives et celles de la Faculté des sciences infirmières de l'Université de Montréal, les auteures analysent les processus qui ont conduit à la formalisation de cette discipline. Elles notent le transfert des savoirs pratiques, que les Soeurs Grises ont acquis comme gestionnaires dans les grands hôpitaux du pays, en savoirs théoriques indispensables à l'établissement d'une science du nursing. Ce faisant, les Soeurs Grises s’engagent également dans un processus de laïcisation du soin, tout en affirmant son lien irrévocable avec les approches catholiques. Ainsi se dégage un modèle de soins qui apparaît comme l'élément structurant de l'organisation hospitalière, et qui résiste aux transformations multiples du champ sanitaire de la période de l'après-première guerre. De plus, en lien avec d'autres travaux, on souligne l'importance de ce modèle et sa persistance dans le système d'éducation et de santé, malgré le mouvement de démocratisation et de laïcisation des années 1970.

This article presents the role of the Grey Nuns in the development of the teaching of nursing in Quebec universities. The authors analyze the processes which have led to the formalization of this discipline through the examination of their archives and those of the Faculty of Nursing Science of the Université de Montréal. They reveal the transfer of practical knowledge that Grey Nuns have acquired as administrators in the major hospitals of the country to theoretical knowledge which is essential to the establishment of nursing as a science. In this manner, the Grey Nuns have also been involved in the process of secularization of health care while confirming their irrevocable tie to Catholic methodologies. Thus emerges a model of care as the structural element of hospital organization resisting the multiple transformations in the health care field in the period following the First World War. Furthermore, in relation to other works, the importance of this model is highlighted as well as its continuance in the educational and health care system in spite of the democratization and secularization movement in the 1970s.

1 Cet article présente certains résultats d'une recherche plus large sur la Faculté des sciences infirmières de l'Université de Montréal dirigée par Yolande Cohen. Nous tenons à remercier le Hannah Institute for the History of Medicine qui a octroyé une bourse de doctorat à Esther Lamontagne. Certaines données sont reprises de sa thèse de doctorat. Nous voulons également remercier Nadia Fahmy-Eid pour sa lecture toujours attentive. 
Au début des années 1920, les Sœurs Grises de Montréal établissent à l'Université de Montréal des cours de formation professionnelle supérieure pour des femmes francophones et catholiques, au moment où les portes des facultés traditionnelles des Arts et Lettres leur sont pratiquement fermées ${ }^{2}$. On pourrait considérer l'entrée de ces femmes à l'université comme un des moments où l'interdit qui pesait sur elles dans le monde de l'éducation supérieure commence à être levé, même si ce n’est que pour une petite minorité d'entre elles. Pourtant, les études sur l'histoire de l'éducation au Québec parlent peu du rôle des Sœurs Grises dans ce processus lent et chaotique que constitue l'entrée des femmes à l'université. Certes, on connaît souvent le nom des premières femmes médecins ou avocates. Mais, que sait-on des premiers grands contingents de femmes qui entrent à l'université et des programmes dits féminins, comme le nursing précisément, dans lesquels elles s'inscrivent? Certains chercheurs qui se sont penchés sur la question de l'éducation des filles et sur l'histoire des professions paramédicales ont montré que cette question est largement dépendante des rapports de pouvoir entre l'État et l'Église et, plus tard, des ordres professionnels dont les femmes restent longtemps exclues. Ainsi, dans leurs travaux, Nadia FahmyEid, Nicole Laurin et Marta Danylewicz ont souligné l'importance des rapports de genre entre les communautés religieuses d'hommes et de femmes. Elles ont aussi mis en évidence des rapports de domination au sein des communautés de femmes, notamment chez

2 Voir Micheline Dumont et Nadia Fahmy-Eid, Les couventines. L'éducation des filles au Québec dans les congrégations religieuses enseignantes, 1840-1960 (Montréal : Boréal, 1986); Nadia Fahmy-Eid (dir.), Femmes, santé et professions : histoire des diététistes et des physiothérapeutes au Québec et en Ontario, 1930-1980 (Montréal : Fides, 1997), 10. 
les hospitalières ${ }^{3}$. Dans ce contexte, Nadia Fahmy-Eid et ses collaboratrices notent l'ambivalence des stratégies déployées par de nombreuses communautés pour développer l'éducation supérieure en diététique et en physiothérapie ${ }^{4}$.

Une sorte de tabou semble planer sur la question de la nature des savoirs féminins (compris comme un ensemble de compétences attribuées aux femmes) dans le système d'éducation. De nombreuses féministes considèrent la question de savoirs propres aux sexes masculin ou féminin comme un essentialisme (ou une naturalisation des savoirs) qu'elles récusent. C'est pourquoi ces dernières hésiteront à inclure le développement des professions féminines comme des victoires féministes. Plus récemment, d'autres féministes ont cherché à souligner la contribution féminine ou maternaliste des mouvements philanthropiques, en particulier ceux qui ont conduit à l'ouverture de véritables bastions féminins dans des institutions qui leur étaient totalement fermées ${ }^{5}$. Le développement d'une éducation supérieure en nursing en est un bon exemple. Comment s'est développé ce processus qui s'inscrit dans l'historiographie de l'éducation des femmes ${ }^{6}$ ? En quoi l'enseignement du nursing à l'université revêt-il une importance particulière pour l'histoire des femmes et pour l'histoire de l'éducation?

Le renouvellement des travaux sur l'histoire du soin introduit d'autres éléments dans l'analyse de la profession infirmière, en particulier en ce qui concerne le rôle de la formation supérieure

3 Dumont et Fahmy-Eid, Les couventines, Marta Danylewicz, Profession religieuse. Un choix pour les Québécoises, 1840-1920 (Montréal: Boréal, 1988), Nicole Laurin, Danielle Juteau et Lorraine Duschesne, À la recherche d'un monde oublié: les communautés religieuses de femmes au Québec de 1900 à 1970 (Montréal: Le Jour, 1991).

4 Fahmy-Eid, Femmes, santé et professions, 79-121.

5 Yolande Cohen, Jacinthe Pepin, Esther Lamontagne et André Duquette, Les sciences infirmières: genèse d'une discipline. Histoire de la Faculté des sciences infirmières de l'Université de Montréal (Montréal: Les Presses de l'Université de Montréal, 2002), Meryn Stuart, "War and Peace: Professional Identities and Nurses' Training, 1914-1930 »: 171-193, dans Elizabeth Smyth et al., eds., Challenging Professions. Historical and Contemporary Perspectives on Women's Professional Work (Toronto: University of Toronto Press, 1999). Voir aussi le texte de Rondalynn Kirkwood, «Blending Vigorous Leadership and Womanly Virtue: Edith Kathleen Russell at the University of Toronto, 1920-1952 ", Bulletin canadien d'histoire de la médecine 11, 1 (1994): 175-206.

6 Dumont et Fahmy-Eid, Les couventines; Nicole Thivierge, Écoles ménagères et Instituts Familiaux: un modèle féminin traditionnel (Québec: IQRC, 1982); LouiseHélène Trottier, " Évolution de la profession infirmière au Québec de 1920 à 1980 » (Mémoire de M.A., Université de Montréal, 1982); Johanne Daigle, « Devenir infirmière: le système d'apprentissage et la formation professionnelle à l'Hôtel-Dieu de Montréal, 1920-1970 » (Thèse de Ph.D., Université du Québec à Montréal, 1990). 
dans le développement professionnel. ${ }^{7}$ Les perspectives de genre, de classe et d'ethnie adoptées dans ces travaux permettent de souligner le rôle des communautés religieuses dans la structuration du système de santé dans les sociétés occidentales à majorité catholique, mais aussi à majorité protestante, et dans la prééminence, en leur sein, de l'institution hospitalière. Des travaux récents nuancent considérablement les analyses qui attribuaient à la seule Florence Nightingale l'implantation du nursing moderne, en donnant davantage de crédit aux communautés religieuses catholiques qui lui ont préparé le terrain ${ }^{8}$.

Dans cette perspective, l'analyse du rôle des Sœurs Grises de Montréal dans le développement d'une formation supérieure en nursing, à laquelle nous nous livrons dans ce texte, permet de mieux comprendre l'ampleur des réformes qu'elles ont initiées, tant au plan local qu'au plan international. Avec les premiers cours supérieurs en nursing à l’Université de Montréal, établis dès 1923, les Sœurs Grises se taillent une place privilégiée dans l'éducation supérieure au Québec. Il en résultera la création d'une faculté de nursing autonome en 1962, tandis que la McGill School of Nursing reste affiliée à la faculté de médecine, comme c'est le cas à l’Université Laval et à l'Université de Toronto.

En même temps, et ce sera le second volet de cet article, ces mêmes Sœurs Grises constituent un ensemble de savoirs et de connaissances pour fonder une nouvelle discipline académique. Quels sont ces savoirs, et en quoi sont-ils liés aux savoirs féminins? Quelles stratégies ont-elles déployées pour les faire reconnaître comme une discipline à part entière? Comment cette brèche ouverte dans le bastion académique a-t-il permis l'établissement d'une nouvelle discipline? Quel rôle les Sœurs Grises jouent-elles dans le

7 Voir entre autres la perspective britannique présentée par Anne-Marie Rafferty, The Politics of Nursing Knowledge (London: Routledge, 1996), la perspective américaine par Barbara Melosh, The Physician's Hand: Work, Culture and Conflict in American Nursing (Philadelphia: Temple University Press, 1982) et Susan Reverby, Ordered to Care: The Dilemma of American Nursing, 1850-1945 (New York: Cambridge University Press, 1987) et la perspective canadienne par Kathryn McPherson, Bedside Matters. The Transformation of Canadian Nursing, 1900-1990 (Toronto: Oxford University Press, 1996) et Yolande Cohen, Profession infirmière. Une histoire des soins dans les hôpitaux du Québec (Montréal: Les Presses de l’Université de Montréal, 2000).

8 Monica Baly, "The Nightingale Nurses : The Myth and the Reality » dans C. Maggs, Nursing History : The State of the Art (London : Croom Helm, 1987) : 33-59 ; Ann Summers, Angels and Citizens : British women as military nurses, 1854-1914 (London: Routledge, 1988); Sioban Nelson, Say Little, Do Much: Nursing, Nuns and Hospitals in the Nineteenth Century (Philadelphia: University of Pennsylvania Press, 2001) ; Pauline Paul, « A History of the Edmonton General Hospital : 1895-1970, « Be Faithful to the Duties of your Calling > » (Ph.D. diss., University of Alberta, 1994). 
développement des sciences infirmières au Québec? Telles sont les questions auxquelles cet article tente d'apporter des réponses à partir de l'étude circonstanciée de l'histoire du nursing à l’Université de Montréal.

Les Sœurs Grises de Montréal font partie d'un groupe plus large de communautés sulpiciennes d'hospitalières, qui comprend, notamment à Montréal, les Sœurs de la Providence et les Religieuses Hospitalières de Saint-Joseph ${ }^{9}$. Comme quelques autres communautés, elles fondent des hôpitaux et oeuvrent au sein d'un important réseau d'institutions au Canada et aux États-Unis. Contrairement à leurs consoeurs de la Providence et de St-Joseph (qui sont restées attachées à un hôpital, l'Hôtel-Dieu), elles ont fondé et pris en charge plusieurs hôpitaux, dans un périmètre qui s'étend à toute l'Amérique du Nord. Ainsi, à Montréal, elles agissent très tôt à l'Hôpital général et assument la gestion de l'Hôpital Notre-Dame (1880). Ailleurs au Québec, elles dirigent l'Hôpital Saint-Roch (1885), l'Hôpital Sainte-Camille (1885), l'Hôpital St-Jean (Haut-Richelieu (1895), l'Hôpital Saint-Paul (1905), l'Hôpital St-Marys (1930) et l'Hôpital Pasteur (1932). Dans l'Ouest canadien, on les retrouve à la tête de grands hôpitaux au Manitoba, en Alberta et en Saskatchewan, et aux États-Unis, dans les États de l'Ohio, du New Jersey et du Massachusetts ${ }^{10}$. Ce vaste ancrage institutionnel différencie les Sœurs Grises des autres groupes hospitaliers qui partagent la pratique de l'accueil, du refuge, des soins et de la formation infirmière. Il doit être donc être considéré comme une motivation importante chez les Sœurs Grises pour développer l'éducation supérieure en nursing.

La gestion des soins infirmiers, plutôt que la pratique soignante elle-même, agit-elle donc comme moteur du développement académique et scientifique en soins infirmiers? Cette hypothèse mérite d'être considérée pour le cas franco-catholique, surtout que l'entrée des infirmières protestantes à l'université s'est produite

9 À la fin du XIX ${ }^{\mathrm{e}}$ siècle, ces trois communautés se partagent une grande part du soin aux malades, agissant dans nombreux asiles, hospices et hôpitaux de la région de Montréal. Voir Micheline D’Allaire, Les communautés religieuses à Montréal. Tome 1 : Les communautés religieuses et l'assistance sociale à Montréal 1659-1900 (Montréal, Éditions du Méridien, 1997).

10 Données tirées de D’Allaire, Les communautés religieuses à Montréal et Soeur Clémentine Drouin, L'Hôpital général des Sœurs de la Charité «Sœurs Grises». Tome III : 1853-1877 (Montréal, Maison mère des Sœurs Grises, 1943), Estelle Mitchell, L'essor apostolique. Histoire de l'hôpital général des Sœurs de la Charité de Montréal "Soeurs Grises ». Tome 4 (1877-1910) (Montréal, Éditions du Méridien, 2000) et L’hôpital Général des Sœurs de la Charité de Montréal "Sœurs Grises ». En toute disponibilité. Tome V : 1910-1935 (Montréal, Éditions continentales, 1996). 
différemment, sous la poussée du mouvement hygiéniste. Ainsi, c'est par les programmes d'hygiène publique que les formations infirmières sont entrées aux Universités McGill, British-Colombia, Toronto, etc. Ce sont d'ailleurs ces mêmes pionnières de l'hygiène qui prennent l'initiative de la formalisation des savoirs infirmiers hospitaliers et rédigent les premiers manuels de nursing en anglais. Mentionnons Bertha Harmer, directrice de l'École de McGill de 1928 à 1934, qui a publié ses premiers travaux, Text-Book of the Principles and Practice of nursing (New York, MacMillan), dès 1922, tandis que le premier manuel de nursing en français de niveau universitaire ne paraît qu'en 1947. De même, l'implantation d'un programme de baccalauréat en nursing à l'Université McGill a lieu en 1957, alors que, dans le réseau catholique, il démarre en 1962. C’est aussi le secteur de la santé publique (plus précisément la santé communautaire) qui donne le ton au mouvement d'institutionnalisation de la recherche scientifique à l'Université McGill, avec son célèbre « McGill Model » développé par Moyra Allen ${ }^{11}$. On ne peut faire ici l'étude de cette différenciation entre les démarches protestantes et catholiques dans les univers académiques. La variable religieuse prend néanmoins tout son sens, croyons-nous. En particulier, peut-on déceler un modèle francocatholique de soins et de savoirs infirmiers lié au pouvoir que les religieuses détiennent dans les institutions hospitalières? À l’Université de Montréal, nous l'avons vu dans un ouvrage récent, ce sont l'administration et l'enseignement des soins infirmiers, puis les spécialités cliniques (les soins post-opératoires par exemple), qui fournissent la base des premières recherches ${ }^{12}$, et ce, même si la première doyenne nommée à la Faculté de Nursing, Alice Girard, provient du secteur de l'hygiène. C'est dire que l'héritage des pionnières marquera les programmes et recherches des universités. Toutes ces questions nous ont conduites à mener notre enquête sur le rôle des Sœurs Grises dans le développement de l'enseignement supérieur en nursing et sur les processus qui les ont menées à élaborer un programme d'études. L'analyse minutieuse des contenus des programmes de formation supérieure en nursing à l'Université de Montréal devrait nous permettre de mieux comprendre le rôle qu’ont joué les Sœurs Grises à la fois dans le développement d'une discipline et dans le déploiement d'un modèle franco-catholique de réforme ou de modernisation du

11 Laurie Gottlieb et Kathleen Rowat, « The McGill Model of Nursing : A Practicederived Model ", Advances in Nursing Science 9, 4 (1987) : 51-61.

12 Cohen et al., Les sciences infirmière : 151-96. 
nursing. Plus que la question de l'accès (relatif) des femmes à l'université, on se demandera quelle logique sous-tendait la mise en œuvre des premiers programmes d'études universitaires exclusivement féminins.

On distinguera deux étapes principales dans ce processus. Durant la première étape, les Sœurs Grises visent à établir la légitimité d'une institution spécifiquement destinée à l'enseignement supérieur du nursing et des programmes de formation professionnelle, avec la création de l'Institut Marguerite d'Youville (IMY) au sein de l'Université de Montréal. L'autre étape consiste à asseoir la crédibilité des savoirs infirmiers et d'un programme propre, dans le contexte d'après-guerre où de nouveaux courants, en santé et en éducation, apparaissent déterminants. Nous appuierons notre analyse sur les archives de l'IMY, dépouillées pour la période durant laquelle Mère Virginie Allaire est aux commandes, soit de 1934 à 1947, et sur l'étude approfondie d'un important manuel de soins infirmiers, rédigé par quatre Sœurs Grises de cet institut.

\section{I- L'institutionnalisation universitaire du nursing : un programme professionnel}

Aux premières heures de la constitution des écoles d'infirmières au tournant du $\mathrm{XX}^{\mathrm{e}}$ siècle au Québec, et malgré les idéologies charitables et féminines qui imprègnent la plupart des pratiques soignantes, les programmes d'études doivent être conformes aux critères établis par la médecine et la chirurgie sur le territoire nord-américain ${ }^{13}$. La formation des infirmières doit ainsi répondre aux besoins d'assistance en techniques et thérapies médicales et chirurgicales. Il s'agit donc de changer les paramètres d'enseignement des soins existants, précédemment mis en place par les infirmières laïques et protestantes du Canada et des États-Unis et par les communautés religieuses. Ces enseignements, souvent donnés au sein des communautés religieuses, visaient la formation spirituelle autant que l'apprentissage des soins. L'éducation personnelle, désignée par Nightingale comme " character training », des notions théoriques et l'apprentissage au chevet du

13 Voir Charles E. Rosenberg, The Care of Strangers: The Rise of America's Hospital System (New York : Basic Books, 1987). Pour le réseau hospitalier catholique, voir André Cellard, $L$ 'histoire de l'Association catholique canadienne de la santé: fidèle à une mission (Ottawa : Association catholique canadienne de la santé, 1990). 
patient formaient la base des enseignements rédigés par des médecins, des soignantes, des Hospitalières et des pionnières du nursing, britanniques, américaines et anglo-canadiennes ${ }^{14}$.

On connaît bien les prescriptions d'assistance au médecin, rédigées par des infirmières et des médecins britanniques et américains, car elles ont été à l'origine des premiers programmes de formation infirmière dans l'univers hospitalier ${ }^{15}$. Inspiré par les idéaux du mouvement hygiéniste, cet ensemble de savoirs a longtemps été considéré comme la base de la réforme des soins infirmiers. Compte tenu des traditions propres au milieu francocatholique, il est intéressant de connaître les fondements des enseignements qui y sont donnés de 1920 à $1947^{16}$. Nous verrons que les Sœurs Grises de Montréal se distinguent d'emblée par leurs préoccupations en faveur d'un enseignement supérieur.

Gestionnaires, on l'a vu plus haut, de plusieurs hôpitaux au Canada et aux États-Unis, les Sœurs Grises constatent l'importance du recrutement d'infirmières qualifiées tant pour pourvoir aux soins que pour enseigner dans les nombreuses écoles d'hôpitaux. Exerçant souvent dans des environnements protestants - dans les provinces canadiennes et les États américains - elles n’hésitent pas à rejoindre des organisations professionnelles (souvent protestantes), acceptent le programme d'agrément proposé par les élites médicales pour réformer les hôpitaux ${ }^{17}$ et investissent dans le perfectionnement de leurs membres les plus douées.

Leurs compétences d'administratrices hospitalières et de soignantes, au Québec et dans les missions canadiennes, sont reconnues. À Montréal, par exemple, les Sulpiciens les appuient et des philanthropes laïcs, qui seront les fondateurs de l'Hôpital Notre-Dame en 1880, les réclament. On requiert aussi les services

14 Les principes de la formation des soignantes de la communauté des Sœurs de la Providence en Amérique du Nord ont été récemment analysés dans Nelson, Say Little, Do Much. En 1859, les Soeurs de la Providence ont d'ailleurs publié un manuel qui s'intitulait: Traité élémentaire de matière médicale et guide pratique des Soeurs de charité de l'Asile de la Providence (Montréal : Eusèbe Sénécal, 1859). Les principes mis de l'avant par les Religieuses Hospitalières de St-Joseph sont décrits dans Daigle, Devenir infirmière, ainsi que dans Cohen, Profession infirmière. En ce qui concerne les Augustines de l'Hôtel-Dieu de Québec, voir François Rousseau, La Croix et le Scalpel. Histoire des Augustines et de l'Hôtel-Dieu de Québec (Québec : Septentrion, 1994). Pour les Sœurs Grises, voir Cohen, Profession infirmière, et Paul, A History of the Edmonton General Hospital.

15 Esther Lamontagne, « Histoire sociale des savoir-faire infirmiers au Québec de 1880 à 1970 » (Mémoire de M.A., Université du Québec à Montréal, 1999).

16 Voir, à ce propos, Cohen, Profession infirmière, 81-102, 209-26.

17 Denis Goulet, Histoire de la Faculté de médecine de l'Université de Montréal, 1843-1993 (Montréal : VLB, 1993), 130-36. 
d’Élodie Mailloux, qui a collaboré à la fondation de l'école de l'Hôpital Saint-Vincent de Toledo en Ohio aux États-Unis, pour fonder la première école d'infirmières francophone au Québec à l'Hôpital Notre-Dame en 1898. C'est d'ailleurs au sein de cet hôpital, affilié à l’Université de Montréal pour la formation des médecins, que le projet de donner aux infirmières une formation universitaire prend forme.

À partir de 1915, l'appui de l'Association des hôpitaux catholiques (AHC) à la réforme des hôpitaux proposée par le Collège américain des chirurgiens en $1914^{18}$ encourage les Sœurs Grises dans leur décision de s'engager dans le perfectionnement de leurs cadres. Dès 1917, les initiatives de la maison mère pour encourager ses membres à assister aux congrès du réseau hospitalier nord-américain et à étudier la question de la formation adéquate des infirmières, et celles de Mère Virginie Allaire, qui fait appel à une universitaire de la Colombie-Britannique, Ethel John ${ }^{19}$, pour donner un cours d'été aux infirmières de Régina en 1919, donnent le coup d'envoi au perfectionnement universitaire au sein de la communauté.

Ainsi, les Sœurs Duckett et Fafard sont-elles sélectionnées pour établir le programme de formation en nursing à l'Université de Montréal. Leur trajectoire est révélatrice. Diplômée de l’École de l'Hôpital Notre-Dame en 1899, puis sa directrice, Sœur Duckett est nommée successivement à des postes de direction dans l'Ouest, à l'Hôpital St-Vincent de Toledo et à l'Hôpital Sainte-Croix, et devient, en 1923, responsable des hôpitaux que gèrent les Sœurs Grises. Quant à Sœur Fafard, elle a obtenu un diplôme de l'École de l'Hôpital Notre-Dame en 1907 et a acquis une spécialisation en pharmacie à l’Université d'Ohio en 1915; puis elle a occupé des postes de direction à Calgary, à Edmonton et à l'Hôpital NotreDame.

18 Cette réforme vient du Rapport Flexner. Déposé en 1910, le rapport souligne la médiocrité de la médecine américaine et " pointe du doigt la piètre qualité de l'enseignement dispensé dans les écoles de médecine de même que la faiblesse des normes hospitalières " . La réception favorable de ce rapport aux Etats-Unis pousse la mise en place, par le Collège américain des chirurgiens en 1914, d’un programme destiné à évaluer le niveau d'excellence des hôpitaux à l'échelle nord-américaine. " Le respect de certaines normes minimales relatives à l'enseignement, au personnel, à l'équipement, etc. vaudrait à l'institution concernée l'octroi d'un agrément > ". L'élite hospitalière catholique se regroupe au sein de l'Association des hôpitaux catholiques en 1915 dans l'optique de soutenir cette réforme. Cellard, L'histoire de l'Association catholique, 10-14. 19 Glennis Zilm et Ethel Warbineck, Legacy : History of Nursing Education at the University of British Columbia (Vancouver : University of British Columbia Press,1994). 
En 1923, les premiers cours supérieurs pour infirmières sont établis à la Faculté de médecine de l’Université de Montréal. Ils sont fréquentés presque exclusivement par les Sœurs Grises. D'autres membres de communautés religieuses hospitalières ${ }^{20}$ et quelques infirmières laïques s'y inscrivent pour parfaire leurs notions d'administration, d'enseignement et de diététique. Pour la première fois, des femmes sont admises dans le réseau universitaire francophone catholique.

Elles suivent un programme de cours spécialisés sanctionné par un certificat. Le programme de 192 heures réparties sur 6 semaines est divisé en deux sections, l'une théorique (3 heures tous les matins) et l'autre pratique (dans les laboratoires et lors des visites dans les hôpitaux et institutions, l'après-midi). Le cours théorique porte sur de nombreux aspects : l'organisation d'une école de gardes-malades (le personnel dirigeant, l'installation ou habitation, les élèves, les registres ou dossiers et les rapports); les différentes formes de services hospitaliers (l’hôpital privé, l’hôpital public, l'hôpital paroissial ou hospice, l'hôpital municipal ou d'isolement); le rôle des gardes-malades dans les services, les dispensaires et les laboratoires; les cours complémentaires (perfectionnement des directrices sur les matières à enseigner dans les écoles d'hôpitaux : anatomie, physiologie, hygiène sociale, chimie, bactériologie et diététique); la vocation et la carrière de garde-malade (formation religieuse sur l'apostolat); la formation éducationnelle et morale d'une garde-malade; les principes généraux de pédagogie (comment appliquer ces principes à l'enseignement médical pour les femmes); l'étiquette professionnelle (honneur professionnel); l'histoire hospitalière et professionnelle. Le cours de diététique, d'une durée de 150 heures, comprend une série de leçons théoriques et pratiques qui s'étendent sur une période de six semaines. Le Dr P. Del Vecchio, spécialiste en alimentation rationnelle, donne le matin une heure de conférence, laquelle est suivie d'une demi-heure de discussion. Les directrices du cours conduisent les heures d'enseignement pratique dans les cuisines de l'Hôpital Notre-Dame. On donne aussi des conférences sur l'organisation du service des diètes dans les hôpitaux ${ }^{21}$.

20 Le terme « hospitalière » désigne les communautés religieuses en milieu hospitalier (ce qui les différencie des congrégations enseignantes), et aussi, plus spécifiquement, la religieuse qui supervise les salles des malades. Ici, on réfère au sens plus large de la communauté.

21 Archives des Sœurs Grises de Montréal (ASGM), fonds L102 1C1, Université de Montréal; Prospectus, Cours de perfectionnement pour les gardes-malades. Diplôme universitaire de garde-malade, Montréal, 1924. 
Cette première expérience n'a duré que deux ans parce qu'interrompue par le décès subi de Sœur Fafard. Elle a toutefois permis deux réalisations : la création d'une revue professionnelle, La Veilleuse ${ }^{22}$, et la poursuite du projet de cours supérieur en soins infirmiers, avec l'appui de l’Université de Montréal. Pour ce projet, la maison mère et l'Université de Montréal retiennent les services de Mère Virginie Allaire, alors supérieure provinciale à Saint-Boniface, afin de créer, établir et assurer la direction d'un nouveau cours qui prendra son envol en 1934 et qui permettra d'établir de façon permanente le nursing à l'université.

De 1928 à 1934, les Sœurs Grises préparent leur programme, recrutent leurs collaboratrices et forment leur personnel. Présentes dans l'institution universitaire et solidement implantées dans le réseau hospitalier catholique canado-américain, elles en utilisent rapidement toutes les ressources pour faire avancer leur projet. Ainsi, membre du Conseil Exécutif de l'Association des hôpitaux catholiques en 1929, Mère Virginie Allaire représente le Canada au comité de rédaction de la revue Hospital Progress, participe à l'établissement de Conférences canadiennes de l'AHC (Ontario, Prairies), et fonde la conférence de Québec en $1932^{23}$.

Grâce à l'appui des médecins de la Faculté de médecine et à la suite d'une négociation menée avec succès auprès de la direction de l'université, les Sœurs Grises fondent en 1934 l'Institut Marguerite d'Youville (YMY), qui sera affilié à l'Université de Montréal en 1936, et y établissent leur premier programme de « sciences hospitalières » sur le modèle professionnel qui allie arts libéraux et matières spécialisées.

Des premiers cours spécialisés établis en 1923, afin de doter les hospitalières d'un certificat universitaire reconnaissant officiellement leur compétence ${ }^{24}$, au programme de baccalauréat en sciences hospitalières créé en 1934, le chemin parcouru est considérable. Les Sœurs Grises adaptent leurs enseignements à la perspective d'une éducation libérale et professionnelle. Elles proposent une année de « matières collégiales » - enseignées en

\footnotetext{
22 Première revue infirmière de type professionnel au Québec, cette revue mensuelle fait la promotion des cours de formation supérieure. Publiée par les infirmières des cours supérieurs (enseignantes, étudiantes et diplômées) et soutenue par le recteur de l’Université de Montréal, Mgr Joseph Vincent Piette, elle est dirigée par les Sœurs Fafard et Duckett. Voir Cohen, Profession infirmière, 164-69.

23 Cellard, L'histoire de l'Association catholique canadienne de la santé, 10-19.

24 ASGM, fonds L102 1C1, Université de Montréal; Prospectus, Cours de perfectionnement pour les gardes-malades.
} 
grande partie par des Sulpiciens ou des Jésuites ${ }^{25}$ - et une année de " matières professionnelles " au sein du programme de baccalauréat, même si l'une et l'autre peuvent mener à un diplôme distinct. Ainsi, une étudiante peut s'inscrire dans un programme d'un an, suivre seulement les matières collégiales ou les matières professionnelles, et recevoir un diplôme de sciences hospitalières.

En inscrivant dans leurs enseignements ces arts libéraux nécessaires à la légitimation d'un programme professionnel à l'université, les Sœurs Grises se conforment aux principes qui guident l'inclusion des formations professionnelles au sein de l'université depuis la deuxième moitié du $\mathrm{XIX}^{\mathrm{e}}$ siècle au Canada. Ces arts libéraux, ces matières « collégiales » comme les appellent les Sœurs Grises, que sont le latin, la géométrie, la philosophie, la rhétorique et la religion, visent à doter les futures professionnelles du soin des connaissances, du langage et du comportement censés gagner la confiance d'une classe bourgeoise et moyenne. Car les Sœurs Grises ont sans doute conscience que les usagers de l'hôpital sont ces classes privilégiées qui peuvent se payer les services professionnels des hospitalières ${ }^{26}$.

Durant l'année de «matières professionnelles », les étudiantes inscrites au baccalauréat en sciences hospitalières à l'Institut Marguerite d'Youville doivent suivre également des cours de sciences pures et biomédicales (chimie alimentaire, physique et chimie, pharmacologie), des cours de sciences humaines (sociologie, histoire), de gestion hospitalière (administration et organisation hospitalières, direction d'école), de pédagogie et d'élocution.

Pour enseigner ces matières dites " professionnelles », la communauté recrute en son sein des enseignantes sélectionnées parmi ses membres les plus compétentes, formées en majorité dans des universités catholiques américaines. L’équipe est composée de six religieuses. Elle compte Sœur St-Louis, qui participe aux enseignements des sciences. Forte d'une expérience de près de 11 ans dans les hôpitaux des Sœurs Grises de l'Ouest canadien, elle excellait, dit-on, comme organisatrice et surveillante des salles d’opération. Elle détient un baccalauréat en sciences de l'éducation des infirmières de l'Université Saint-Louis. Sœur Barry, qui

\footnotetext{
25 Annuaires de l'IMY, 1936 à 1948.

26 Voir, à ce propos, Rosenberg, The Care of Strangers; Burton J. Bledstein, The Culture of Professionalism. The Middle Class and the Development of Higher Education in America (New York: W.W. Norton and Co., 1976); A.B. McKillop, Matters of Mind: The University in Ontario 1791-1951 (Toronto : University of Toronto Press, 1994).
} 
enseigne les sciences hospitalières, possède le même profil : 11 ans d'expérience de direction, de supervision et d'enseignement dans les hôpitaux des Sœurs Grises, notamment en Nouvelle-Angleterre, suivis d'un baccalauréat en sciences de l'éducation des infirmières de l'Université Saint-Louis. Sœur Coderre, qui enseigne la diététique, a cumulé les formations d'infirmières, d'enseignement ménager, d'agriculture et de diététique, et a occupé les postes de supérieure, pharmacienne et directrice. En plus d'enseigner à l'IMY, elle est diététicienne à l'Hôpital Notre-Dame. Sœur Denise Lefebvre, qui se joindra à l'équipe en 1939, possède pour sa part un baccalauréat ès arts, un baccalauréat en sciences de l'éducation des infirmières de l'Université St-Louis et une licence en nursing de l'Université catholique de Washington. Sur Sœur Marie-Rose Lacroix, qui enseigne les sciences, on possède peu d'informations, si ce n'est qu'elle a dirigé l'école d'infirmières de St-Jean-surRichelieu à partir de 1925 et qu'elle détient un baccalauréat ès arts, auquel elle ajoutera une maîtrise en lettres ${ }^{27}$.

Mère Virginie Allaire, fondatrice et directrice de l'Institut Marguerite d'Youville de 1934 à 1947, mise donc sur un programme professionnel et sur l'expertise pratique et théorique de ses consoeurs pour établir le nursing comme discipline enseignée à l’Université de Montréal. Les Sœurs Grises réussissent à faire de leur programme en sciences hospitalières le passage obligé pour la formation de la grande majorité des hospitalières des hôpitaux franco-catholiques : sur 238 diplômées en sciences hospitalières en 1955, les SG ont non seulement formé de nombreuses membres de leur propre communauté (34,5 \%), mais aussi des laïques (31,1\%) et plusieurs religieuses appartenant à d'autres communautés $(34,4$ $\%)^{28}$. Ces mêmes diplômées occupent les postes d'hospitalières (40 $\%)$, d'enseignantes (16\%), de directrices d'écoles d'infirmières, et de directrices et d'assistantes directrices du nursing $(13 \%)^{29}$. La formation supérieure en nursing connaît donc un véritable succès

\footnotetext{
27 ASGM, Notices biographiques..

28 Les plus nombreuses sont les Religieuses Hospitalières de Saint-Joseph (23 \%), suivies par les Sœurs Grises de la Croix (12 \%), les Sœurs Grises de St-Hyacinthe (8\%). D’autres communautés hospitalières présentes sur le territoire du Québec et des communautés missionnaires constituent le reste du contingent (56 \%) : ce sont les Sœurs de la miséricorde, les Filles de la sagesse, les Filles de la charité du Sacré-Cœur, les Religieuses Hospitalières d’Arthabaska, les Sœurs de la Providence, les Augustines de Chicoutimi, de Québec et de Lévis, les Religieuses de l'Espérance, les Missionnaires Notre-Dame des Anges, les Oblates franciscaines de Saint-Joseph, les Missionnaires du Christ-Roi, les Petites franciscaines de Marie et les Sœurs de la Providence de Kingston. ASGM, Statistiques de l'Institut Marguerite d'Youville, 1955.

29 ASGM, Statistiques de l'Institut Marguerite d'Youville, 1955.
} 
auprès des communautés religieuses qui saisissent l'occasion de doter leurs membres de diplômes supérieurs leur permettant d'occuper des postes de direction.

Ainsi, les Sœurs Grises ont réalisé la première étape de leur ambitieux projet, celle de l'institutionnalisation d'une formation supérieure en nursing à l'Université de Montréal. Elles ont fait de leur programme un incontournable pour la formation en nursing de toutes les communautés hospitalières franco-catholiques au Québec. Elles doivent cependant affronter des groupes d'intérêt puissants et des spécialités biomédicales en pleine mutation. Ainsi, ayant réussi à intégrer au programme de nursing une formation en gestion et éducation hospitalières, les Sœurs Grises tentent aussi d’inclure l'option diététique dans le programme. Leur projet suscite l'opposition de la direction de l'université. Rapidement, cette spécialité n’apparaît plus comme relevant de leurs compétences et devient, en 1941, une discipline universitaire enseignée à la Faculté de médecine de l’Université de Montréal.

Doit-on pour autant faire des distinctions entre les éléments techniques et scientifiques de la démarche du soin? Quand et comment certaines connaissances et pratiques sortent-elles du giron du nursing? L'introduction des sciences humaines dans l'enseignement du nursing marque-t-elle, comme le souligne Nadia Fahmy-Eid, un niveau plus avancé dans l'avènement du care $e^{30}$ ?

C'est ce que nous tenterons de comprendre en examinant la seconde étape de leur projet qui consiste à développer des savoirs infirmiers propres aux enseignements en nursing. De 1934 à 1947, les Sœurs Grises s'engagent dans la formalisation des savoirs infirmiers, une démarche qui aboutira, à plus longue échéance, à la désacralisation et la dénaturalisation de l'exercice infirmier. En effet, la diminution et même l'absence marquée de références à la mission apostolique et féminine des infirmières caractérisent cette nouvelle approche.

\section{II- La formalisation des savoirs infirmiers}

À la fin de leur programme, les candidates au baccalauréat en sciences hospitalières doivent produire un travail de recherche dirigé. Les titres des thèses et travaux de recherches produits par les étudiantes de l’Institut Marguerite d’Youville entre 1934 et 1946 
$(40 \mathrm{au} \text { total })^{31}$ indiquent les thèmes privilégiés par les professeurs et les infirmières. Antérieures au développement de la recherche scientifique universitaire en nursing qui s'amorce à la fin des années 1960, ces données confirment les orientations du nursing autour de trois thèmes principaux : la formation infirmière, l'administration de l'hôpital et les soins aux malades.

La formation infirmière dans le système hospitalier inspire près de la moitié des travaux réalisés. Le fonctionnement d'une école s'avère l'objet principal des recherches : on tente de comprendre et de mieux connaître le système hospitalier francophone et catholique. Le système de formation infirmière est comparé aux autres systèmes canadiens et analysé de façon systématique : cours préliminaires, formation technique, formation en salle d'opération, formation théorique, enseignement clinique, etc. On constate ainsi à travers ces travaux que la formation devient avec le temps de plus en plus spécialisée : on distingue la formation technique en nursing, en salle d'opération, en assistance médicale, en hygiène, etc. Dans les années 1940, les études portent davantage sur l'enseignement clinique dans les départements de l'hôpital ou dans les secteurs de santé publique.

Le rôle administratif et de direction de l'hospitalière ${ }^{32}$ dans la distribution des soins suscite aussi beaucoup d'intérêt chez les futures bachelières. Dans leurs travaux, le caractère religieux de la pratique des hospitalières transparaît. L'alimentation fait aussi l’objet de quelques travaux pendant la période où les hospitalières enseignaient la diététique, soit avant 1941.

Le soin et ses composantes techniques, thérapeutiques, morales et spirituelles, et son objet, le malade hospitalisé, alimentent une recherche continue durant toute la décennie 1940. Ainsi, il apparaît clairement que les Sœurs Grises calquent le contenu de la formation qu'elles donnent sur le rôle qu'elles occupent dans les hôpitaux. Une formation générale pour l'administration et l'enseignement infirmiers au niveau universitaire est, selon elles, la clé qui leur permettra de renforcer la place des infirmières à l'université et dans le système de santé. Toutefois, leur apport original réside dans la formalisation de leur conception particulière des soins. C'est ce que l’on cherchera maintenant à comprendre.

\footnotetext{
31 ASGM, Liste des thèses et travaux de recherche - décrite comme incomplète, 1966.

32 Le terme " hospitalière " utilisé ici prend le sens spécifique de la position hiérarchique de l'infirmière-religieuse au sein des services infirmiers.
} 
Le soin des malades : principes et techniques

La volonté de formaliser les savoirs infirmiers n'est certes pas propre à l'Institut Marguerite d'Youville. Les articles parus dans les revues La Veilleuse (1924-1927) et La garde-malade canadienne-française créée en 1928 ainsi les publications des hospitalières et des médecins au sein de leurs institutions témoignent de cette tendance qui se généralise au Québec ${ }^{33}$. Cependant, le statut institutionnel que les Sœurs Grises ont acquis dans la formation supérieure des infirmières leur permet de concentrer leurs efforts dans le domaine de l'éducation supérieure.

Le manuel Le soin des malades. Principes et techniques, dont la rédaction est amorcée en 1944 et terminée en 1947 par les Sœurs Denise Lefebvre (à l'aube de sa nomination au poste de directrice de l'IMY), Adèle Levasseur, Germaine Dessureau et Flore Bellemare, illustre ce développement. Ce manuel de 814 pages, tiré à 4800 exemplaires $^{34}$ et dédié sans surprise à Mère Virginie Allaire, " sagace artisane de progrès dans le domaine hospitalier ${ }^{35}$, présente la synthèse des recherches sur les soins hospitaliers et leur gestion ${ }^{36}$. Les références scientifiques nationales et internationales abondent. Les auteures citent en effet des articles rédigés dans l'American Journal of Nursing par des infirmières américaines, des textes d'infirmières du réseau francophone et catholique publiés dans les revues La garde-malade canadienne française, le Bulletin des infirmières catholiques du Canada et dans le livre des Sœurs de la Charité de Québec, ainsi que des textes de Bertha Harmer (directrice de l’École de nursing McGill de 1928 à 1934) et d'autres infirmières canadiennes du réseau anglo-protestant dans The Canadian Nurse. Le manuel fait aussi état de nombreux articles des revues Modern Hospital, Hospital Progress et The Canadian Hospital et réfère également aux travaux des médecins français Rudaux, Gley, Collet et Hédon $^{37}$, et à ceux de l’infirmière française Léonie Chaptal ${ }^{38}$.

33 Soeurs de la Providence, Traité élémentaire de matière, Sœur Allard, Principes élémentaires concernant le soin des malades (Montréal : Hôtel-Dieu de Montréal, 1931).

34 L'ensemble des exemplaires sont vendus en 1953. ASGM, IMY, Rapport Annuel, 1947 à 1953.

35 Institut Marguerite d'Youville (IMY), Le soin des malades : Principes et techniques (Montréal : IMY, 1947).

36 ASGM, fonds L102/B1, pièce 20.1.

37 P. Rudaux, Précis élémentaire d'anatomie, de physiologie et de thérapeutique appliquée (Paris : Masson et Cie, 1936), E. Gley, Traité élémentaire de physiologie, $9^{\mathrm{e}}$ édition (Paris : J.B. Baillère et fils, 1938), T.J. Collet, Précis de pathologie interne (Paris : G. Doin et Cie, 1931), E. Hédon, Précis de physiologie, $12^{\mathrm{e}}$ édition (Paris : G. 
Enfin, des références aux travaux des médecins montréalais G. Hébert et L.K. Ferguson ${ }^{39}$ et aux articles du Laval médical sont également présentes. De fait, le manuel s'appuie sur un large éventail de sources.

À l'instar des autres congrégations franco-catholiques, les Sœurs Grises réitèrent dans ce manuel leur conception religieuse du soin, vu comme le soulagement des misères humaines pour l'amour du Christ. Elles s'en distinguent toutefois par l'intégration de trois autres éléments empruntés aux infirmières du réseau anglophone et laïque (notamment de l'Américaine Isabel Hampton $^{40}$ et de la Canadienne Bertha Harmer). D'abord, elles définissent les principes fondamentaux, moraux, physiques, intellectuels et religieux de l'exercice infirmier, qui régissent les soins et le comportement des soignantes. Ensuite, elles réaffirment l'importance de la " technique nursing " dont elles avaient déjà esquissé les contours à la fin des années 1920. Dans leur évocation des principes et des techniques, transparaît la relation humaine soignant / soigné, avec la constante préoccupation des besoins des malades. C'est cette combinaison de principes et de pratiques qui rend la production des Sœurs Grises originale et qui constitue les bases multiples du savoir infirmier dans les institutions francophones et catholiques. Cette approche est contemporaine du mouvement en faveur de la jonction, voire la subordination de l'acte de foi, de la piété, à l'exercice pratique et scientifique du soin à autrui chez les communautés religieuses, une perspective que les praticiennes laïques ont conceptualisée plus tard à travers la notion de soin holistique ${ }^{41}$.

\section{Les principes du savoir infirmier}

Explicitement et contrairement aux écrits de la période qui précède 1920, les Sœurs Grises précisent les qualités nécessaires à l'exercice infirmier et en font un objet d'apprentissage et de

\footnotetext{
Doin et Cie, 1939).

38 L. Chaptal, Le livre de l'infirmière (Paris : Masson et cie, 1928).

39 G. Hébert, Formulaire de l'Hôpital Notre-Dame, Montréal, 1937 ; L. K. Ferguson, Care of Surgery of Ambulatory Patient (Montreal : J.B. Lippincott, 1942).

40 A ce sujet, voir Cohen et al., Les sciences infirmières, 57.

41 Robert Wuthnow, Learning to Care: Elementary Kindness in an Age of Indifference (New York : Oxford University Press, 1995); Sioban Nelson, A Genealogy of Care of the Sick. Nursing Holism and Pious Practice (Southsea : Nursing Praxis International, 2000); Verna Benner Carson and Harold G. Koenig, Parish Nursing Stories of Service and Care (Philadelphia : Templeton Foundation Press, 2002).
} 
compétence. Ainsi, les principes fondamentaux qui sous-tendent l'acte du soin consistent à consoler et à soulager l'être humain souffrant. En formulant ainsi ces principes, les Sœurs Grises opèrent une rupture radicale avec la vision féminine essentialiste et charitable du soin. Ces notions ne sont donc plus considérées comme allant de soi pour toutes les femmes, compte tenu des « qualités naturelles » de ces dernières et ne peuvent plus être des caractéristiques personnelles ${ }^{42}$. Les Soeurs Grises rendent également publics et objectifs les critères qu'elles utilisent dans le processus d'évaluation des postulantes ${ }^{43}$. Des considérations d'ordre moral, physique, intellectuel et religieux colorent toutefois les compétences requises. On constate que certaines qualités morales et physiques relèvent du discours courant sur la mystique de la femme soignante.

Les qualités morales (sympathie, bonté, grandeur d’âme, générosité de sentiments, désintéressement, compassion, dévouement, gaieté, honnêteté et loyauté, obéissance et bonne volonté à être dirigée et évaluée sur la technique de soin et ses résultats) sont mises au service de la relation de soin. Les malades sont au centre de cette relation, et la qualité de cette relation dépend des rapports entre les soignants. La hiérarchie hospitalière est comprise selon le principe d'une relation de service de l'infirmière envers son malade. Elle semble soutenue par la compassion et le dévouement, valeurs empruntées à une supposée nature féminine, mais aussi à celles des professions libérales, comme le droit ou la médecine. Le désintéressement, la bonté, l'honnêteté et la loyauté sont autant de qualités qui sont requises chez les membres des professions libérales (les médecins et les avocats par exemple) qui veulent servir une classe moyenne en expansion ${ }^{44}$, notamment au XIX ${ }^{\mathrm{e}}$ siècle, lors de l'implantation de leurs formations au sein de l'université.

Les qualités physiques (santé, culture physique, délicatesse des sens, manières avenantes, expression, dextérité manuelle) traduisent leur volonté de rendre efficace leur geste de prise en charge du patient. L'apparence, le geste et la posture semblent ici significatifs de la perception que les malades ont de la capacité de l'infirmière à les rassurer et à se charger de leur souffrance.

42 Exprimé ainsi : " Don naturel que des femmes possèdent », " carrière digne d'une élite féminine ». IMY, Le soin des malades, 4.

43 Lamontagne, " Histoire sociale des savoir-faire infirmiers au Québec ».

44 Bledstein, The Culture of Professionalism, Rosenberg, The Care of Strangers, McKillop, Matters of Mind. 
D’ailleurs, les Sœurs Grises précisent dans leur manuel l'importance de l'élégance de la démarche et du geste qui donnent « de l'aplomb au maintien, de l'assurance, qualités essentielles à une infirmière ${ }^{45}$. Elles soulignent l'importance du contrôle des émotions, de l'usage de la fermeté, de la douceur ainsi que de la clarté de l'expression verbale. La relation de " prise en charge » apparaît ici fondamentale à l'exercice du soin. La douceur y est déterminante et, comme le souligne l'historienne Kathryn McPherson, c'est ici qu'on peut dégager un rapport clair avec le discours courant sur la féminité ${ }^{46}$.

Pour les religieuses, les qualités intellectuelles (un jugement sûr, un esprit vif et observateur, une bonne mémoire, du tact) servent à acquérir et à utiliser les informations qui guideront le soin. Ces qualités seront nourries par les notions théoriques acquises des disciplines biomédicales, humaines et sociales. L'intégration de ces connaissances multidisciplinaires sert à l'évaluation des besoins du malade par l'infirmière. Ce n'est qu'après cette évaluation que les exercices techniques (de confort et d'entretien, par exemple), thérapeutiques ou de soutien moral doivent être exécutés.

Enfin, les qualités religieuses donnent un sens au travail de l'infirmière, permettent de l'ancrer dans un cadre plus large d'une aide à l'humanité souffrante et d'en imprégner sa relation avec le malade. Les Sœurs Grises vont jusqu'à dire que « la pensée de la présence de Dieu en elle-même et en autrui " ${ }^{47}$ s'observe physiquement, en donnant aux traits des infirmières un reflet de sérénité. Plus spécifiquement, ce savoir-être d'inspiration religieuse envers le malade s'inscrit dans une conception générale du soin qu'elles se sont engagées à donner aux autres, plus démunis ou malades. Les religieuses hospitalières épousent, dans leur travail de nursing, les principes éthiques de l'Église catholique et respectent la morale catholique ${ }^{48}$. Dans le cas des Soeurs Grises, la tradition de Mère d'Youville inspire la pratique infirmière comme le ferait un exercice de charité : en soignant le malade, la religieuse soigne aussi son Seigneur Jésus-Christ. Ces qualités religieuses s'inscrivent dans le discours qui prescrit aux femmes, même si elles sont religieuses, les tâches de soin. Mais ces mêmes principes catholiques qui cantonnent les femmes aux tâches de soin

IMY, Le soin des maladies, 6.

McPherson, Bedside Matters, 164-204.

IMY, Le soin des maladies, 7.

Paul, A History of the Edmonton General Hospital, 449. 
permettent, dans le secteur professionnel, l'émergence d'un principe infirmier. La reconnaissance de l'être humain comme une entité physique et spirituelle par l'Église catholique conduit à la nécessité du soin spirituel (soin des âmes, sanctification des âmes) et du soin des corps (par la science pratique ${ }^{49}$. Les religieuses considèrent alors la personne comme un tout et s'y dévouent. Aussi, Pauline Paul désigne-t-elle l'approche des religieuses dans le soin comme une approche holistique ${ }^{50}$. Enseignant aux étudiantes laïques le dévouement aux malades selon les principes de l'Église catholique, les religieuses établissent le cadre d'un soin holistique en milieu hospitalier.

La « technique nursing »

Les travaux de Sœur Allard de l'Hôtel-Dieu de Montréal introduisent au début des années $1930^{51}$ de nouvelles techniques et procédures de soins, dans un effort de distinguer le nursing des pratiques hétéroclites présentes dans les écrits des années précédentes. Dans leur manuel, Principes élémentaires concernant le soin des malades, les Sœurs Grises reprennent certaines de ces procédures (de soins de confort, d'entretien, prophylactiques et thérapeutiques) afin de systématiser les tâches et de les uniformiser. Trois procédures, l’une générale, les deux autres plus précises, sont ainsi proposées. Les infirmières doivent prendre en considération une série d'éléments lors de chaque soin : «1confort du patient, 2-propreté et bonne apparence, 3- économies de temps, d'effort et de matière première, 4- technique et habileté, 5fidélité aux détails, 6- effets thérapeutiques, 7- simplicité et assurance dans le travail ${ }^{52}$. L'attitude personnelle dans la relation du patient doit se transformer en rituel de soin : «1- Dire bonjour et sourire, 2- Esprit de prévision, être à l'écoute, se rappeler des besoins, 3- Ne pas lui annoncer de mauvaises nouvelles, 4Confidentialité, 5- Éloges du médecin, 6- Pas de bruit, 7- Tenue irréprochable, 8- Assister les invalides pour les repas, faire

Ibid., 416.

Ibid., 450.

On doit à Sœur Allard la rédaction d'une chronique intitulée « Technique » dans la GMCF à partir de 1928 et la publication, en 1931, du manuel Principes élémentaires concernant le soin des malades.

52 Allard, Principes élémentaires, 9. 
attention à la propreté et l'apparence de leur cabaret ${ }^{53}$ ». Dans ce processus, Sœur Allard considérait la technique du soin des malades comme « l'habile maniement du malade avec le moins de malaise possible; l'habile maniement des objets stérilisés sans danger de les contaminer; l'exclusion des mouvements inutiles de manière à assurer le maximum de vitesse compatible avec la plus grande habileté ${ }^{54}$. L'habileté, l'efficacité et l'économie caractérisent ainsi ces rituels.

La systématisation des soins infirmiers implique la transformation des tâches d'assistance en une procédure très ritualisée et balisée qui définit la nature de l'attention portée au patient. Malgré la complexification des appareils utilisés et l'augmentation des prescriptions médicales ${ }^{55}$, la procédure des soins vise ainsi à conférer à l'acte infirmier une nouvelle efficacité et une règle d'ordre. Avec l'énumération de consignes précises et la codification des tâches (ou gestes) de soin, les Sœurs Grises considèrent la relation de soin entre l'infirmière et le malade comme relevant d'un service pour lequel l'infirmière doit être formée et rémunérée même si le dévouement religieux guide aussi son action.

Les directives sont claires. L’infirmière sera évaluée sur la rapidité des actes posés, sur les étapes qu'elle a suivies et les moyens adoptés pour mener le patient à la guérison. On tiendra également compte de l'économie de temps, d'effort et de matériel, et de l'apparence soignée et finie de son travail. Lors du cours

53 Sœur Allard, « Technique. Moyen de procurer confort physique et moral au patient ", La Garde-Malade Canadienne-Française, mars 1929, 182.

54 Allard, Principes élémentaires, 9.

55 Les innovations technologiques surviennent à un rythme de plus en plus rapide, la situation sanitaire change, les maladies infectieuses tendent à disparaître, tandis que d'autres prennent de l'ampleur (maladies cardio-vasculaires, allergies...). La recherche dans le domaine de la pharmaceutique procure aux médecins l'antibiothérapie (pénicilline, streptomycine, etc.) pour traiter les maladies infectieuses. Des médicaments comme la cortisone, de nouveaux diurétiques, des bêtabloquants utilisés en cardiologie, de la cimétidine pour traiter les ulcères, l'insuline pour traiter les diabétiques, les neuroleptiques utilisés pour le soin des maladies mentales, et d'autres encore apparaissent. La chirurgie est aussi l’une des spécialités qui connaît un développement impressionnant (chirurgie de la douleur dans les années 1940, trépanation en neurochirurgie, transplantations, chirurgie traumatologique lors de la Deuxième Guerre mondiale, chirurgie plastique, etc.). La vaccination a aussi permis de prévenir beaucoup de maladies, particulièrement la poliomyélite, la tuberculose, la rubéole et la diphtérie. De plus, l'évolution des techniques de diagnostic a fait faire un pas de géant à la médecine. Plusieurs appareils permettent de faire des examens et des diagnostics précis de l'état d'un patient (électrocardiographe, électroencéphalographe et des techniques d'examens telles la radiologie, la xérographie, le lavement baryté, le scanner, etc.). Voir l'ouvrage de François Guérard, Histoire de la santé au Québec (Montréal : Boréal Express, 1996). 
pratique, un intérêt particulier est porté au confort et à la satisfaction du patient, au fait qu'il ne souffre pas ou peu et que lui ou son entourage soient moins inquiets. De nombreux exemples tirés de la section «Problèmes et questions » du manuel Le soin au malade (1947) illustrent cette préoccupation. Ainsi, la question de l'humiliation d'un malade est soulevée : « Une malade, dans une salle commune, souffre de pédiculose et en est fort humiliée : dicter la conduite de l'infirmière à son égard $»^{56}$. Selon les directives du manuel, l'infirmière devrait inviter la malade à surmonter son humiliation pour pouvoir être traitée en toute confiance. C'est surtout par l'usage de tact, de paroles d'encouragement favorisant la gaieté, de discrétion, que l'infirmière peut « donner regain et fraîcheur à l'existence de la malade ». L’infirmière devra considérer l'histoire (histoire familiale, milieu de vie, etc.) de la malade pour saisir plus justement ses comportements (ici d’humiliation) et y remédier. Une fois l'humiliation disparue ou atténuée, et le traitement réussi (soins hygiéniques de la chevelure et destruction des parasites), elle pourra lui donner des conseils d'hygiène, toujours en usant de tact et en s'adaptant au milieu, à l'âge et à l'éducation de la malade.

Plusieurs autres exemples signalent l'importance du rôle de l'infirmière dans le soulagement ou le réconfort d'un malade : « Une personne (est) hospitalisée en chambre privée, et, par suite d'un revers de fortune, elle doit être transférée en chambre publique. Quels sentiments peuvent alors la troubler et quelle sera votre manière d'agir avec cette personne? " ${ }^{57}$; "Quelles inquiétudes avez-vous remarqué (sic) chez un trachéotomisé? Comment le rassurer? ${ }^{58}$; " Vous vous apercevez que des visiteurs fatiguent le malade, comment y remédier? $\aleph^{59}$. Ce qui apparaît d'abord comme des règles de comportement pour alléger la souffrance ou l'inconfort d'un patient, souvent reliées au discours courant sur la féminité, peut également être compris comme une forme élaborée de gestion de l'environnement humain et social au sein de l'hôpital. Si le comportement de l'infirmière doit servir le bien-être du patient, il doit également ordonner l'ensemble du milieu hospitalier qui l'entoure. En fait, l'ensemble de cette codification sert non seulement à définir le nouveau profil de

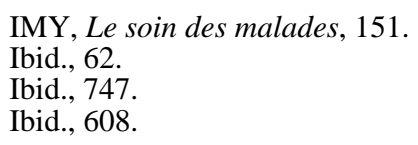


l'infirmière professionnelle, à établir ses connaissances sur des savoirs et des techniques précises, mais aussi à en faire le pivot des nouvelles normes de gestion hospitalière. Toutes ces qualités deviennent dans les écrits des Sœurs Grises des compétences qui s'acquièrent uniquement dans les différents services hospitaliers, qui font l'objet d'une évaluation en bonne et due forme, et qui fondent l'expertise reconnue de l'infirmière. La relation au malade en est un des fondements, tout comme la volonté de rationaliser les soins et de les rendre plus techniques. L’infirmière est au service du malade, mais aussi au centre de l'administration hospitalière, ce que l'on ne s'étonnera pas de trouver chez les Sœurs Grises qui ont acquis leur propre compétence comme gestionnaires d'hôpitaux et enseignantes dans leurs écoles.

C'est sans doute ce qui constitue la particularité de la contribution de cette communauté au nursing. Les Sœurs Grises n'hésitent pas à inscrire leur vision des soins infirmiers dans le courant nord-américain de réforme hospitalière, et se distinguent ainsi de bien des hospitalières catholiques qui se méfiaient de la mainmise des groupes laïcs et protestants dans ce mouvement. Elles n'adhèrent toutefois pas aux solutions préconisées par ces derniers en ce qui concerne notamment les fondements des savoirs enseignés en nursing. Elles se distinguent aussi nettement du modèle anglo-protestant où l'absence d'expérience antérieure des infirmières laïques dans les grands hôpitaux a conduit les réformateurs à penser le nursing comme le prolongement de l'hygiène. Les Sœurs Grises élaborent ainsi leur propre modèle de soin, qui vise à développer une formation supérieure pour moderniser le nursing et en faire l'élément structurant de l'organisation hospitalière. À ce titre, on a souvent critiqué cette vision en la qualifiant d'élitiste, car elle entrevoit le rôle de l'infirmière, comme celui de l'hospitalière, au centre de tout l'édifice du soin, dans des hôpitaux qui sont leur chasse gardée. Ce qui apparaît à travers cette étude des premiers manuels de soins infirmiers, c’est l'importance accordée par les Sœurs Grises à l'hôpital comme lieu central pour prodiguer des soins à la population.

\section{Conclusion}

L'Institut Marguerite d'Youville, avec ses activités d'enseignement et de recherche, prodigue, comme à l'École supérieure de nursing de l'Université McGill, un enseignement 
libéral professionnel. Ce qui apparaît à la lumière de cette étude, c'est que l'IMY est également un laboratoire où s'élaborent des conceptions du nursing qui allient plusieurs expériences et expertises, qui semblent uniques aux Sœurs Grises. Cet ensemble de connaissances constituera la base des programmes et enseignements spécialisés des écoles de nursing et de la recherche universitaire dans le secteur franco-catholique durant les années $1970^{60}$.

À ce titre, les Sœurs Grises participent au mouvement de réforme hospitalière nord-américain, qui préconise que les soins infirmiers soient laïcs et placés sous la direction des médecins. Leur engagement dans ce mouvement les a conduites à concevoir d'une façon nouvelle les soins infirmiers, pour y inclure des aspects techniques et scientifiques, ce qui leur a permis ensuite de développer, comme leurs consoeurs américaines et canadiennes, une formation supérieure à l'université pour les infirmières francocatholiques. Que les hospitalières des communautés religieuses aient été les premières à en bénéficier ne fait aucun doute. Avec la création d'une institution d'éducation supérieure, l'Institut Marguerite d'Youville, et leur participation à la fondation de la Faculté de nursing de l’Université de Montréal, les Sœurs Grises ont néanmoins fait plus : elles ont ouvert une brèche durable dans le système universitaire pour toutes les femmes laïques à la recherche d'une carrière. Au-delà de l'institution, qu'elles ont dû quitter dans la foulée de la démocratisation et de la laïcisation de l'enseignement supérieur, elles ont laissé une marque encore plus durable avec la formalisation originale des savoirs en nursing, savoirs que l'on retrouve encore dans les programmes de formation offerts par la faculté de nursing dans les années 1970.

Cette conception particulière du nursing développée par les Sœurs Grises s’inscrit dans un processus de désacralisation et de dénaturalisation de l'exercice infirmier, une conception que reprendront sans peine les infirmières laïques et qui dominera la scène de l'éducation supérieure en nursing au tournant des années 1960. Ainsi, alors que la loi des infirmières est modifiée en 1969 pour admettre des hommes à la profession et que le mouvement de démocratisation des institutions de santé et d'éducation met fin à la domination religieuse dans ces secteurs, la production des savoirs infirmiers se poursuivra en continuité avec la trame établie par le processus de formalisation des savoirs dans les manuels 
infirmiers. Les sciences humaines et sociales viendront remplacer les explications religieuses et morales (souvent naturelles) qui s'effritent dans les années d'après-guerre. Ainsi, les écrits de Hildegard Peplau, Interpersonal Relations in Nursing (1952), et ceux de Virginia Henderson sur les besoins fondamentaux des malades ${ }^{61}$ sont déjà présents dans les programmes et travaux à l’Université de Montréal durant les décennies 1960 et 1970.

Sans transgresser ouvertement les limites du genre (sphère du soin attribuée aux femmes) et les rapports apparents de domination entre les sexes, les Sœurs Grises ont néanmoins réussi à traduire dans un nouveau corps de savoirs leur conception des soins infirmiers, comme compétence et base d'une expertise propre. Leur expérience d'administratrices et d'enseignantes se retrouve également dans leurs visions des soins, traduisant in fine la pratique unique des hospitalières franco-catholiques.

61 Ces travaux ont été évoqués par Esther Lamontagne : "Savoirs infirmiers et professionnalisation ", communication présentée au Colloque Enjeux et promesses de l' histoire des femmes, UQAM, Montréal, 30 novembre 2001. 